\title{
Reynolds stresses and one-dimensional spectra for a vortex model of homogeneous anisotropic turbulence
}

\author{
D. I. Pullin \\ Graduate Aeronautical Laboratories 105-50, California Institute of Technology, Pasadena, California \\ 91125 \\ P. G. Saffman \\ Applied Mathematics 217-50, California Institute of Technology, Pasadena, California 91125
}

(Received 24 June 1993; accepted 30 December 1993)

Homogeneous anisotropic turbulence consisting of a collection of straight vortex structures is
considered, each with a cylindrically unidirectional, but otherwise arbitrary, internal vorticity
field. The orientations of the structures are given by a distribution $P$ of appropriate Euler angles
describing the transformation from laboratory to structure-fixed axes. One-dimensional spectra
of the velocity components are calculated in terms of $P$, and the shell-summed energy spectrum.
An exact kinematic relation is found in which volume-averaged Reynolds stresses are
proportional to the turbulent kinetic energy of the vortex collection times a tensor moment of $P$.
A class of large-eddy simulation models for nonhomogeneous turbulence is proposed based on
application of the present results to the calculation of subgrid Reynolds stresses. These are
illustrated by the development of a simplified model using a rapid-distortion-like approximation.

\section{INTRODUCTION}

It is well known that the statistical kinematics of isotropic turbulence, by which we refer to, for example, relations between the shell-summed energy spectrum and the various one-dimensional spectra, can be developed in complete generality, without the need for specific information concerning the structure of the underlying vorticity field (e.g., Batchelor ${ }^{1}$ ). This is apparently not the case for an equivalent kinematic theory of homogeneous but anisotropic turbulence, where, in general, some properties of the vorticity structure must be known in order to derive kinematic relations.

In the present paper we study the kinematics of a model of homogeneous anisotropic turbulence based on the assumption that the small-scale vorticity consists of a superposition of vorticity fields or "structures," each with the property that the vorticity is unidirectional, with no explicit dependence of the vorticity magnitude on the coordinate parallel to vortex lines (Saffman and Pullin ${ }^{2}$ ). A typical realization is a locally rectilinear vortex with an arbitrary cross-sectional vorticity distribution embedded in a three-dimensional, spatially uniform velocity-gradient field (whose components may be time dependent), produced by larger-scale structures. Such vortices may be characterized as "two dimensional," although this term is misleading, as it suggests that no stretching or rotation of vortex lines by the large-scale field is allowed, which is not the case for the present model. Evidence that structures of this type are characteristic of the small scales of isotropic turbulence is provided by the numerical simulations of Kerr, ${ }^{3}$ Ashurst et al., ${ }^{4}$ Vincent and Meneguzzi, ${ }^{5}$ and others, based in part on the observed tendency for alignment between the vorticity vector and the eigenvector corre- sponding to the algebraically intermediate principal rate of strain. It has been shown by Lundgren ${ }^{6}$ that a statistically homogeneous field of strained spiral vortices that exhibit this property can have a $k^{-5 / 3}$ inertial range, where $k=|\mathbf{k}|$ is the modulus of the wave number. Pullin and Saffman ${ }^{7}$ have shown that this model can be used to calculate the higher-order statistics of the velocity-gradient field.

In Sec. II, we specify the required vortex structure in a general way and define the anisotropy in terms of probability density functions (PDF) of the orientations of the structures relative to 1aboratory axes. An expression for the Reynolds stress tensor is obtained by intuitive argument in Sec. III. This result is used in Sec. IV in the formulation of a class of large-eddy simulation models based on the idea of local subgrid homogeneity for the unresolved subgrid scales, and on a model equation, giving the response of the orientation PDF to the fields of the velocity and the velocity-gradient tensor of the resolved, nonhomogeneous large-eddy flow. A feature of these models is that the vorticity distribution inside the subgrid structures need not be explicitly specified or calculated, provided one is prepared to invoke Kolmogorov scaling for the inertial/dissipationrange subgrid energy spectrum. When this is done, a model containing two numerical free parameters is obtained. These are the Kolmogorov constant and the skewness or dimensionless cubic velocity derivative. In Sec. V, relations for one-dimensional energy spectra in terms of the shellsummed energy spectrum are derived, and it is shown that from these, a formal proof of the Reynolds-stress result of Sec. III can be obtained. An alternative proof is given in Appendix A, while Appendix B demonstrates that the large-eddy simulation models discussed in Sec. IV should 
reduce to the fully resolved Navier-Stokes equations when the local Kolmogorov length scale is larger than the local spatial resolution.

\section{VORTEX STRUCTURE}

\section{A. Cylindrically unidirectional vorticity}

We take axes $\left(r_{1}, r_{2}, r_{3}\right)$ with corresponding unit vectors $\left(\mathbf{e}_{1}, \mathbf{e}_{2}, \mathbf{e}_{3}\right)$ fixed in space. The homogeneous turbulence consists of a superposition of velocity fields from a large collection of straight vortex structures. The orientation of a particular structure will be defined by unit vectors $\left(\mathbf{e}_{1}^{\prime}, \mathbf{e}_{2}^{\prime}, \mathbf{e}_{3}^{\prime}\right)$, with $\mathbf{e}_{3}^{\prime}$ parallel to the vorticity in the structure, i.e.,

$$
\omega=\omega\left(r_{1}^{\prime}, r_{2}^{\prime}, t\right) \mathbf{e}_{3}^{\prime},
$$

where $\left(r_{1}^{\prime}, r_{2}^{\prime}, r_{3}^{\prime}\right)$ are coordinates in vortex-fixed axes with $r_{1}^{\prime}, r_{2}^{\prime}$, lying in the plane of the cross section in the directions $\mathbf{e}_{1}^{\prime}, \mathbf{e}_{2}^{\prime}$. We shall refer to vorticity distributions like (1), which show no dependence on $r_{3}^{\prime}$, as cylindrically unidirectional. The function $\omega\left(r_{1}^{\prime}, r_{2}^{\prime}, t\right)$ is arbitrary, but is assumed to be compact. Nonexclusive examples may include

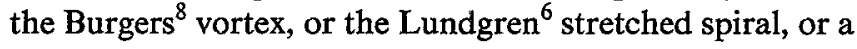
distribution corresponding to some complex fractal set. It will later be seen that the precise details of $\omega$ appear in the final result only through the shell-summed energy spec- trum. An essential assumption is that any interactions between structures of different orientation, to be described subsequently, or between the large scales and the structures maintains the cylindrical unidirectionality of (1). Thus, for example, vortex stretching, compression, or tilting is allowed, but the twisting of vortex lines associated with axial flow internal to the structures is not. Otherwise $\omega\left(r_{1}^{\prime}, r_{2}^{\prime}, t\right)$ may be supposed to locally satisfy the NavierStokes equations, although we note that the present results are essentially kinematic, and vortex/Navier-Stokes dynamics of the fine scales will only enter our arguments in an implicit way.

We denote by $E_{i j}$ the rotation matrix describing coordinate transformations between the $\left(r_{1}, r_{2}, r_{3}\right)$ and the $\left(r_{1}^{\prime}, r_{2}^{\prime}, r_{3}^{\prime}\right)$ axes systems. This is defined, such that a vector $v_{i}$ and a tensor $T_{i j}$ are transformed as $v_{j}=E_{i j} v_{i}^{\prime}$ and $T_{i j}$ $=E_{p i} T_{p q}^{\prime} E_{q j}$, respectively. Except where otherwise specified, the Einstein summation convention will be used. The components of $E_{i j}$ are expressible in terms of the Euler angles, $\alpha, \beta$, and $\gamma$, describing rotation of the $\left(r_{1}, r_{2}, r_{3}\right)$ axes to the $\left(r_{1}^{\prime}, r_{2}^{\prime}, r_{3}^{\prime}\right)$ axes, where $\alpha$ is colatitude (the angle between the $r_{3}$ and $r_{3}^{\prime}$ axes), $\beta$ is longitude, and $\gamma$ is spin about the $r_{3}^{\prime}$ axis (see, e.g., Jeffreys and Jeffreys ${ }^{9}$ Sec. 4.034). These are

$$
\mathbf{E}=\left(\begin{array}{ccc}
\cos \alpha \cos \beta \cos \gamma-\sin \beta \sin \gamma & \cos \alpha \sin \beta \cos \gamma+\cos \beta \sin \gamma & -\sin \alpha \cos \gamma \\
-\cos \alpha \cos \beta \sin \gamma-\sin \beta \cos \gamma & -\cos \alpha \sin \beta \sin \gamma+\cos \beta \cos \gamma & \sin \alpha \sin \gamma \\
\sin \alpha \cos \beta & \sin \alpha \sin \beta & \cos \alpha
\end{array}\right)
$$

\section{B. Probability density of the Euler angles}

We now utilize the the probability density function $P(\alpha, \beta, \gamma)$ of the Euler angles introduced by Saffman and Pullin $^{2}$ and defined such that $P \sin \alpha d \alpha d \beta d \gamma / 8 \pi^{2}$ is the probability that $\left(\mathbf{e}_{1}^{\prime}, \mathbf{e}_{2}^{\prime}, \mathbf{e}_{3}^{\prime}\right)$ lie in the range $\alpha$ to $\alpha+d \alpha, \beta$ to $\beta+d \beta$, and $\gamma$ to $\gamma+d \gamma$. In general, $P(\alpha, \beta, \gamma)$ is expected to be determined by the interaction between large scales and the vortex structures, and we will return to this problem in Sec. IV. The expectation of any function $f\left(E_{i j}\right)$ averaged over the orientations of the structures is

$$
\begin{aligned}
\left\langle\left\langle f\left(E_{i j}\right)\right\rangle\right\rangle= & \frac{1}{8 \pi^{2}} \int_{0}^{\pi} \int_{0}^{2 \pi} \int_{0}^{2 \pi} f\left(E_{i j}\right) P(\alpha, \beta, \gamma) \\
& \times \sin \alpha d \alpha d \beta d \gamma .
\end{aligned}
$$

The function $P(\alpha, \beta, \gamma)$ describes the anisotropy of the turbulence. If $P=1$, the turbulence is isotropic. We will denote by $\hat{P}(\alpha, \beta)$ the probability density function of the Euler angles in the special case when $P$ is independent of $\gamma$, and define the single angle bracket average over Euler angles as

$$
\begin{aligned}
\left\langle f\left(E_{i j}\right)\right\rangle= & \frac{1}{8 \pi^{2}} \int_{0}^{\pi} \int_{0}^{2 \pi} \int_{0}^{2 \pi} f\left(E_{i j}\right) \hat{P}(\alpha, \beta) \\
& \times \sin \alpha d \alpha d \beta d \gamma .
\end{aligned}
$$

\section{REYNOLDS STRESSES}

It is our aim to calculate the Reynolds stress tensor $\overline{\overline{u_{i} u_{j}}}$ at a point in the fluid, where $u_{i}$ is the instantaneous velocity in a fixed reference frame in which the mean velocity is zero, and the double overbar denotes an ensemble average or a volume average over a large box containing the turbulence. We will use double angle brackets to denote averages over the orientations in the sense of (3) and a single overbar to denote an average over the $r_{1}^{\prime}-r_{2}^{\prime}$ crosssectional plane of a single structure. We make the identification

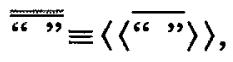

and assume that averages over the structure cross sections are independent of the Euler angles. 
It is now assumed that $P$ is independent of the spin angle $\gamma$, that is, at fixed $\alpha, \beta$, which give the direction of the vorticity, all values of spin are equally likely. This is natural physically since the vorticity within a structure will tend to impart rotation. With this restriction we give the following plausibility argument for the calculation of $\overline{\overline{u_{i} u_{j}}}$. Consider structures with $\mathrm{e}_{3}^{\prime}$ in $d \alpha d \beta$. In the primed axis system, the Reynolds stresses produced by these structures, when averaged over the structure cross section and over the spin angle $\gamma$, must be given by

$$
\overline{u_{i}^{\prime} u_{j}^{\prime}}=\overline{\left(u_{m}^{\prime}\right)^{2}} Z_{i j} \text {, }
$$

where $Z_{i j}$ is a diagonal tensor with diagonal elements $\left(\frac{1}{2}\right.$, $\left.\frac{1}{2}, 0\right)$. This is because the velocity field associated with the structures, with mean square velocity $\overline{\left(u_{m}^{\prime}\right)^{2}}$, must lie in the $r_{1}^{\prime}, r_{2}^{\prime}$ plane, and so when averaged over $\gamma$, the off-diagonal components will vanish and the turbulence energy will be equally divided between the $r_{1}^{\prime}$ and $r_{2}^{\prime}$ directions. Upon transforming to $\left(r_{1}, r_{2}, r_{3}\right)$ axes and averaging over the remaining Euler angles, we find

$$
\overline{\overline{u_{i} u_{j}}}=\overline{\overline{u_{m}^{2}}}\left\langle E_{p i} Z_{p q} E_{q j}\right\rangle,
$$

where we note that the components of $E_{p i} Z_{p q} E_{q j}$ (i.e., before integration over any of the Euler angles),

$$
\mathbf{E}^{\mathrm{T}} \cdot \mathbf{Z} \cdot \mathbf{E}=\left(\begin{array}{ccc}
\frac{1}{2}\left(1-\sin ^{2} \alpha \cos ^{2} \beta\right) & -\frac{1}{4} \sin ^{2} \alpha \sin 2 \beta & -\frac{1}{4} \sin 2 \alpha \cos \beta \\
-\frac{1}{4} \sin ^{2} \alpha \sin 2 \beta & \frac{1}{2}\left(1-\sin ^{2} \alpha \sin ^{2} \beta\right) & -\frac{1}{4} \sin 2 \alpha \sin \beta \\
-\frac{1}{4} \sin 2 \alpha \cos \beta & -\frac{1}{4} \sin 2 \alpha \sin \beta & \frac{1}{2}\left(1-\cos ^{2} \alpha\right)
\end{array}\right)
$$

are independent of $\gamma$.

Using the standard result for homogeneous turbulence, $\overline{\overline{u_{m}^{2}}}=2 \int_{0}^{\infty} E(k) d k$, where $E(k)$ is the shell-summed energy spectrum and $k=|\mathbf{k}|$, we can now obtain

$$
\overline{\overline{u_{i} u_{j}}}=2 \int_{0}^{\infty} E(k) d k\left\langle E_{p i} Z_{p q} E_{q j}\right\rangle \text {. }
$$

Equation (8) is our principal result. It is easily verified that when $P=1$, (8) gives $\overline{\overline{u_{i} u_{j}}}=\frac{1}{\overline{3}} \overline{\overline{u_{m}^{2}}} \delta_{i j}$, as required for isotropic turbulence. If the turbulence is decaying, then $\overline{u_{m}^{2}}$, and perhaps $\hat{P}$, will be functions of time. In Sec. $\mathrm{V}$ and in Appendix A, formal derivations of (8) are given and it is shown that this is an exact kinematic result for homogeneous anisotropic turbulence with the vortex structure specified in Sec. II.

\section{A CLASS OF LARGE-EDDY SIMULATION MODELS}

\section{A. Equations for the resolved scales}

We consider the large-eddy simulation of the NavierStokes equations on a grid with typical cell size $O(\Delta \mathrm{x})$ and time step $O(\Delta t)$. While acknowledging the conceptual problems associated with the derivation of self-consistent equations for this purpose, and, in particular, terms in the form of "Leonard" stresses, which we have omitted (see Leonard ${ }^{10}$ and Germano et al. ${ }^{11}$ for a discussion of this problem), we nonetheless utilize the following large-eddy simulation equations:

$$
\begin{aligned}
& \frac{\partial \widetilde{U}_{i}}{\partial x_{i}}=0, \\
& \frac{\partial \widetilde{U}_{i}}{\partial t}+\frac{\partial}{\partial x_{j}}\left(\widetilde{U}_{i} \widetilde{U}_{j}\right)=-\frac{\partial \tilde{P}}{\partial x_{i}}-\frac{\partial T_{i j}}{\partial x_{j}}+v \frac{\partial^{2} \widetilde{U}_{i}}{\partial x_{j} \partial x_{j}},
\end{aligned}
$$

where $U_{i}(\mathbf{x}, t)$ is the velocity, $P$ is the pressure, $v$ is the kinematic viscosity, and

$$
T_{i j}=\widetilde{u_{i} u_{j}} \text {. }
$$

We remark that in (11) we have defined $T_{i j}$ with a positive sign on the right-hand side for later convenience in relating this quantity to the two-point velocity correlation tensor. Equations (9)-(10) may be obtained heuristically by first decomposing the velocity field as $U_{i}(\mathbf{x}, t)=\widetilde{U}_{i}(\mathbf{x}, t)+u_{i}$ $(\mathrm{x}, t: \tau)$, where the overtilde, by definition, refers to supergrid or resolved scale quantities and $u_{i}(\mathbf{x}, t: \tau)$ are subgrid scale motions on the fast time scale $\tau$, and then by taking a volume-time average over a discretized region of $\mathrm{x}-t$ space of size $O(\Delta \mathbf{x} \Delta t)$. In (10) and (11), $T_{i j}$ are the subgrid Reynolds stresses arising from motions on scales smaller that $\Delta x$. We have used $\mathbf{x}$ to denote supergrid coordinates and will use $\mathbf{r}$ to denote cell-centered subgrid coordinates.

\section{B. Subgrid Reynolds stresses}

We now introduce a wave number cutoff $k_{c}=2 \pi / \Delta x$. The subgrid stresses are modeled by first assuming that the subgrid turbulence is locally homogeneous, so that $T_{i j}$ can be equated to the right-hand side of $(8)$, with the lower limit of integration replaced by $k_{c}$,

$$
T_{i j}=2 \int_{k_{c}}^{\infty} E(k) d k\left\langle E_{p i} Z_{p q} E_{q j}\right\rangle .
$$

The shell-summed energy spectrum $E(k)$ can be estimated by invoking subgrid dynamics in the form of a model for (1) [see (48) or (A8) of Appendix A], for example, the Lundgren ${ }^{6}-$ stretched spiral vortex. The test of such a calculation would certainly be that it gives agreement with experiment, perhaps in the form of Kolmogorov and inertial-range scaling for $E(k)$. Accepting this and noting 
that the detailed vorticity distribution appears in (12) only through $E(k)$, we can sidestep dynamical issues if we are prepared to assume first that $k_{c}$ lies in the inertial subrange, and second, that it is reasonable to use a Kolmogorov-type model for $E(k)$ directly. Put another way, (12) gives a point of contact between Reynolds stresses produced by local anisotropy and the phenomenology of $E(k)$ in the inertial and dissipation ranges. Thus, whatever $\omega(r, \theta, t)$ is present in the vortex core, evolving according to local dynamics, we tacitly assume that it will produce an $E(k)$ [see (48) or (A8); note, in particular, that $E(k)$ given by (48) is independent of $P$, agreeing with experiment. We therefore proceed by making a choice for $E(k)$ based on Kolmogorov scaling as

$$
E(k)=\mathscr{K}_{0} \epsilon^{2 / 3} k^{-5 / 3} e^{-C k \eta}, \quad k_{c}<k,
$$

where $\mathscr{K}_{0}$ is the Kolmogorov prefactor, $\epsilon$ is the local total dissipation, $\eta=\left(v^{3} / \epsilon\right)^{1 / 4}$ is the local Kolmogorov length, and $C$ is a constant. Other choices, as suggested by physical or numerical experiment or as calculated by subgrid dynamics, would serve equally well, but we use (13) presently for simplicity.

Equation (13) contains parameters $\mathscr{K}_{0}$ and $C$, which can be related via the Kármán-Howarth equation to the skewness $S_{3}$ for isotropic turbulence using equation (7.5.15) of Batchelor ${ }^{1}$ as

$$
S_{3}=-\left(\frac{160}{3 C^{8 / 3} \mathscr{K}_{0} \Gamma\left(\frac{4}{3}\right)}\right)^{-1 / 2},
$$

where $\Gamma(\cdots)$ is the gamma function. The choice $\mathscr{K}_{0}=2.0, C=6$, gives $S_{3}=-0.50$. Using (13) in (12) gives

$$
T_{i j}=2 C^{2 / 3} \cdot \mathscr{K}_{0} \epsilon^{2 / 3} \eta^{2 / 3} \Gamma\left(-\frac{2}{3}, C k_{c} \eta\right)\left\langle E_{p i} Z_{p q} E_{q j}\right\rangle,
$$

where $\Gamma(\mu, z)=\int_{z}^{\infty} v^{\mu-1} e^{-v} d v$ is the incomplete gamma function.

\section{Response of the subgrid scales}

To obtain closure we must now relate $\epsilon$ and $\hat{P}(\alpha, \beta)$ to the supergrid velocity field $\widetilde{\mathbf{U}}(\mathbf{x}, t)$ and velocity gradient tensor $\partial \widetilde{U}_{i} / \partial x_{j}$. Since $k_{c}$ lies in the inertial range, then the local flux of turbulent energy to scales smaller than $2 \pi / k_{c}$ should be independent of wave number and determined by local production. It is then reasonable to assume that the difference between $\epsilon$ and the supergrid viscous dissipation is in balance with the local supergrid turbulent energy production, which is the rate of working of the supergrid strain against the subgrid Reynolds stresses,

$$
\epsilon=2 v\left(\widetilde{S}_{i j}\right)^{2}-\tilde{S}_{i j} T_{i j}
$$

where $\widetilde{S}_{i j}=\frac{1}{2}\left(\partial \widetilde{U}_{i} / \partial x_{j}+\partial \widetilde{U}_{j} / \partial x_{i}\right)$ is the supergrid rate-ofstrain tensor. Using (15) then gives

$$
\begin{aligned}
\epsilon= & 2 v\left(\tilde{S}_{i j}\right)^{2}-2 C^{2 / 3} \mathscr{K}_{0} \epsilon^{2 / 3} \eta^{2 / 3} \Gamma\left(-\frac{2}{3}, C k_{c} \eta\right) \\
& \times\left\langle E_{p i} Z_{p q} E_{q j}\right\rangle \tilde{S}_{i j} .
\end{aligned}
$$

In the limit $v \rightarrow 0$ with $\epsilon$ fixed, (15) becomes

$$
T_{i j}=\frac{3 \mathscr{K}_{0} \epsilon^{2 / 3}}{k_{c}^{2 / 3}}\left\langle E_{p i} Z_{p q} E_{q j}\right\rangle,
$$

and (16) then has the solution

$$
\epsilon=\frac{27 \mathscr{K}_{0}^{3}}{k_{c}^{2}}\left(-\left\langle E_{p i} Z_{p q} E_{q j}\right\rangle \bar{S}_{i j}\right)^{3} \text {. }
$$

Thus

$$
T_{i j}=\frac{27 \mathscr{K}_{0}^{3}}{k_{c}^{2}}\left(\left\langle E_{l s} Z_{l m} E_{m r}\right\rangle \tilde{S}_{s r}\right)^{2}\left\langle E_{p i} Z_{p q} E_{q j}\right\rangle,
$$

from which it follows that $T_{i j}$ scales as $\Delta x^{2}$. An alternative route to (20) is found by putting $C=0$ in (13).

In order to obtain an equation for $\hat{P}(\alpha, \beta ; \mathbf{x}, t)$, we need to address the central problem of the interaction between the large scales, represented here by the supergrid field, and the subgrid vortex structures. We suppose that the subgrid structures are convected by the supergrid velocity $\widetilde{U}_{l}$ and at the same time are subject to, in a frame of reference moving with the local supergrid velocity, rotation and stretching (or compression) by the supergrid velocitygradient tensor $\partial \widetilde{U}_{i} / \partial x_{j}$. Since we would expect that any viable dynamical or phenomenological model for $E(k)$, $k>k_{c}$ must already have included, either explicitly in a dynamical model or implicitly for a phenomenological model, the process of vortex stretching by the larger scales $k<k_{c}$ [for example, the inertial range present in (13) requires the implicit assumption that $\epsilon$ is independent of $v$, which, in turn, implies stretching of the fine scales], then we need only consider convection and rotation effects of the supergrid field. We may then write

$$
\frac{\partial \hat{P}}{\partial t}+\widetilde{U}_{j} \frac{\partial \hat{P}}{\partial x_{j}}+\frac{1}{\sin \alpha} \frac{\partial}{\partial \alpha}\left(\widetilde{U}_{\alpha} \sin \alpha \hat{P}\right)+\frac{1}{\sin \alpha} \frac{\partial}{\partial \beta}\left(\widetilde{U}_{\beta} \hat{P}\right)=0
$$

where $\widetilde{U}_{\alpha}, \widetilde{U}_{\beta}$ are the projections of the local supergrid field $r_{j} \partial \widetilde{U}_{i} / \partial x_{j}$ onto the unit sphere. The third and fourth terms of (21) give the contribution to the rate of change of $\hat{P}$ from rotation by $\partial \widetilde{U}_{i} / \partial x_{j}$. When appropriate boundary conditions for $\hat{P}$ are specified, (21) gives closure. The resultant model is not of the eddy-viscosity type. It is perhaps too complicated for the purposes of computation (since $\hat{P}$ is a function of five independent variables plus time), but is presented for completeness and as a possible starting point for further work on the class of models discussed here.

While it may be possible to construct from (21) suitable transport equations for the required $\left\langle E_{p i} Z_{p q} E_{q j}\right\rangle$ moments of $\hat{P}$, we propose as an alternative a very simple model that does not use (21) directly, but is motivated by a rapid-distortion approximation based on the idea that the subgrid structures respond on a fast time scale to the supergrid strain. If it is assumed that the rotation terms of (21) arc dominant over the convection term, then it is easy to show that asymptotically, for large time, vortices will become aligned with the directions in space corresponding to the orientation of the attracting critical points of the $\left(\widetilde{U}_{\alpha}, \widetilde{U}_{\beta}\right)$ vector field on the unit sphere. These directions 
may be found from an analysis ${ }^{12}$ of the $3 \times 3$ eigensystem corresponding to the $\partial \widetilde{U}_{i} / \partial x_{j}$ tensor. We simplify the problem by considering only the symmetric part of $\partial \widetilde{U}_{i} / \partial x_{j}$. Let the local eigenvectors of $\widetilde{S}_{i j}$ be $\left(\mathbf{e}_{1}^{s}, \mathbf{e}_{2}^{s}, \mathbf{e}_{3}^{s}\right)$, with corresponding eigenvalues or principal rates of strain $\left(\lambda_{1}, \lambda_{2}, \lambda_{3}\right)$, such that $\lambda_{1}+\lambda_{2}+\lambda_{3}=0$, and ordered as $\lambda_{1}<\lambda_{2}<\lambda_{3}$. When the eigenvalues are distinct, the critical points associated with $\left(\mathbf{e}_{1}^{s}, \mathbf{e}_{2}^{s}, \mathbf{e}_{3}^{s}\right)$ are a source-like node, a saddle point, and a sink-like node, respectively. If we estimate that the fraction of time that a structure spends in the neighborhood of $\mathbf{e}_{2}^{s}$ before moving toward $\mathbf{e}_{3}^{s}$ is $\max \left\{0, \lambda_{2}\right\} /\left|\lambda_{1}\right|$, then we can approximate that, in our rapid distortion model, the fraction of local subgrid structures tending to become aligned with $\mathbf{e}_{3}^{s}$ is $\lambda_{3} /\left(\max \left\{0, \lambda_{2}\right\}+\lambda_{3}\right)$, and with $\mathbf{e}_{2}^{s}$ is $\max \left\{0, \lambda_{2}\right\} /$ $\left(\max \left\{0, \lambda_{2}\right\}+\lambda_{3}\right)$. This says that, on the average, the subgrid structures will become aligned with the directions of the local extensional principal rates of strain of the supergrid field. When calculated in the frame of reference defined by the unit vectors $\left(\mathbf{e}_{1}^{s}, \mathbf{e}_{2}^{s}, \mathbf{e}_{3}^{s}\right)$ (the superscript $s$ refers to this frame), the moments $\left\langle E_{p i}^{s} Z_{p q} E_{q j}^{s}\right\rangle$ are then

$$
\begin{aligned}
\left\langle E_{p i}^{s} Z_{p q} E_{q j}^{s}\right\rangle= & \frac{\lambda_{3}}{\max \left\{0, \lambda_{2}\right\}+\lambda_{3}}\left(\begin{array}{ccc}
\frac{1}{2} & 0 & 0 \\
0 & \frac{1}{2} & 0 \\
0 & 0 & 0
\end{array}\right) \\
& +\frac{\max \left\{0, \lambda_{2}\right\}}{\max \left\{0, \lambda_{2}\right\}+\lambda_{3}}\left(\begin{array}{ccc}
\frac{1}{2} & 0 & 0 \\
0 & 0 & 0 \\
0 & 0 & \frac{1}{2}
\end{array}\right),
\end{aligned}
$$

from which the $\left\langle E_{p i} Z_{p q} E_{q j}\right\rangle$ in the fixed axes can be calculated. In particular, from $(22)$ and $\widetilde{S}_{i j}^{s}=\operatorname{diag}\left[\lambda_{1}, \lambda_{2}, \lambda_{3}\right]$, the contraction $\left\langle E_{p i} Z_{p q} E_{q j}\right\rangle \widetilde{S}_{i j}$ in (17) is given by

$$
-\left\langle E_{p i} Z_{p q} E_{q j}\right\rangle \widetilde{S}_{i j}=\frac{1}{2}\left(\frac{\max \left\{0, \lambda_{2}\right\}^{2}+\lambda_{3}^{2}}{\max \left\{0, \lambda_{2}\right\}+\lambda_{3}}\right),
$$

and is positive. This guarantees that $\epsilon$ is positive in the model. It is shown in Appendix B that the above model reverts to the Navier-Stokes equations at the supergrid resolution when $\eta$ is large compared to the cell size $\Delta x$. It is straightforward to construct alternative rapid-distortion models based on the eigensystem corresponding to the full $\partial \widetilde{U}_{i} / \partial x_{j}$ tensor, but these are more complex than (22)(23).

\section{ONE-DIMENSIONAL SPECTRA}

\section{A. Kinematics}

We calculate one-dimensional velocity spectra in terms of $k_{3}$, the component of $\mathbf{k}$ in the three-direction. We begin with the two-point velocity and vorticity correlation tensors for homogeneous anisotropic turbulence,

$$
\begin{aligned}
& R_{i j}(\rho)=\overline{\overline{u_{i}(\mathbf{r}) u_{j}(\mathbf{r}+\boldsymbol{\rho})}} \\
& W_{i j}(\rho)=\overline{\overline{\omega_{i}(\mathbf{r}) \omega_{j}(\mathbf{r}+\boldsymbol{\rho})}},
\end{aligned}
$$

where $\rho$ is the separation. These are related to the cncrgy and vorticity spectrum tensors through

$$
\begin{aligned}
& \boldsymbol{R}_{i j}(\boldsymbol{\rho})=\int_{-\infty}^{\infty} \int_{-\infty}^{\infty} \int_{-\infty}^{\infty} \Phi_{i j}(\mathbf{k}) e^{i \mathbf{k} \cdot \boldsymbol{\rho}} d k_{1} d k_{2} d k_{3}, \\
& W_{i j}(\boldsymbol{\rho})=\int_{-\infty}^{\infty} \int_{-\infty}^{\infty} \int_{-\infty}^{\infty} \boldsymbol{\Omega}_{i j}(\mathbf{k}) e^{i \mathbf{k} \cdot \boldsymbol{\rho}} d k_{1} d k_{2} d k_{3},
\end{aligned}
$$

and

$$
\boldsymbol{\Phi}_{i j}(\mathbf{k})=\frac{1}{8 \pi^{3}} \int_{-\infty}^{\infty} \int_{-\infty}^{\infty} \int_{-\infty}^{\infty} R_{i j}(\rho) e^{-i \mathbf{k} \cdot \boldsymbol{\rho}} d \rho_{1} d \rho_{2} d \rho_{3},
$$

$\Omega_{l j}(\mathbf{k})$

$$
=\frac{1}{8 \pi^{3}} \int_{-\infty}^{\infty} \int_{-\infty}^{\infty} \int_{-\infty}^{\infty} W_{i j}(\boldsymbol{\rho}) e^{-i \mathrm{k} \cdot \rho} d \rho_{1} d \rho_{2} d \rho_{3} .
$$

The one-dimensional spectrum tensor is defined by

$$
\Theta_{i j}\left(k_{3}\right)=\frac{1}{2 \pi} \int_{-\infty}^{\infty} R_{i j}\left(0,0, \rho_{3}\right) e^{-i k_{3} \rho_{3}} d \rho_{3},
$$

which, using (26) and (28), may be shown to be equal to

$$
\Theta_{i j}\left(k_{3}\right)=\int_{-\infty}^{\infty} \int_{-\infty}^{\infty} \Phi_{i j}(\mathbf{k}) d k_{1} d k_{2} .
$$

The Reynolds-stress tensor $R_{i j}(0)$ is given by

$$
\begin{aligned}
R_{i j}(0) & =\int_{-\infty}^{\infty} \Theta_{i j}\left(k_{3}\right) d k_{3} \\
& =\int_{-\infty}^{\infty} \int_{-\infty}^{\infty} \int_{-\infty}^{\infty} \Phi_{i j}(\mathbf{k}) d k_{1} d k_{2} d k_{3} .
\end{aligned}
$$

From (31) and the relation between $\Phi_{i j}(\rho)$ and $\Omega_{i j}(\rho)$,

$$
\Omega_{i j}(\mathbf{k})=\boldsymbol{\Phi}_{q q}(\mathbf{k})\left(k^{2} \delta_{i j}-k_{i} k_{j}\right)-k^{2} \boldsymbol{\Phi}_{j i}(\mathbf{k}),
$$

where $k^{2}=k_{1}^{2}+k_{2}^{2}+k_{3}^{2}$ may be obtained,

$$
\begin{aligned}
\Theta_{i j}\left(k_{3}\right)= & \int_{-\infty}^{\infty} \int_{-\infty}^{\infty} \frac{1}{k^{2}}\left[\Omega_{q q}(\mathbf{k})\left(\delta_{i j}-\frac{k_{i} k_{j}}{k^{2}}\right)\right. \\
& \left.-\Omega_{j i}(\mathbf{k})\right] d k_{1} d k_{2} .
\end{aligned}
$$

Using (1) to express the vorticity in $\left(r_{1}, r_{2}, r_{3}\right)$ coordinates as

$$
\omega_{i}^{(m)}(\mathbf{r}, t)=E_{3 i} \omega^{(m)}\left(r_{1}^{\prime}, r_{2}^{\prime}, t\right),
$$

we may write, for $W_{i j}(\rho)$,

$$
\begin{aligned}
W_{i j}(\rho)= & \frac{1}{L^{3}} \sum_{m} l_{m} \int_{-\infty}^{\infty} \int_{-\infty}^{\infty} \int_{0}^{m} \int_{0}^{2 \pi} \int_{0}^{2 \pi} \\
& \times \omega^{(m)}\left(r_{1}^{\prime}, r_{2}^{\prime}, t\right) \omega^{(m)}\left(r_{1}^{\prime}+\rho_{1}^{\prime}, r_{2}^{\prime}+\rho_{2}^{\prime}, t\right) E_{3 i} E_{3 j} \\
& \times P(\alpha, \beta, \gamma) d r_{1}^{\prime} d r_{2}^{\prime} \sin \alpha d \alpha d \beta d \gamma
\end{aligned}
$$

where the sum is over all structures, denoted individually by the superscript $(m)$, which are in the box of volume $L^{3}$ containing the turbulence at time $t, l_{m}$ is the instantaneous 
length of a structure, and $\left(\rho_{1}^{\prime}, \rho_{2}^{\prime}\right)$ is the projection of $\rho$ on the $\left(r_{1}^{\prime}-r_{2}^{\prime}\right)$ plane. Introducing the sectional Fourier transforms,

$$
\begin{aligned}
\hat{\omega}^{(m)}\left(\kappa_{1}, \kappa_{2}\right)= & \frac{1}{4 \pi^{2}} \int_{-\infty}^{\infty} \int_{-\infty}^{\infty} \omega^{(m)}\left(r_{1}^{\prime}, r_{2}^{\prime}, t\right) \\
& \times e^{-i \kappa_{1} r_{1}^{\prime}-i \kappa_{2} r_{2}^{\prime}} d r_{1}^{\prime} d r_{2}^{\prime}, \\
\omega^{(m)}\left(r_{1}^{\prime}, r_{2}^{\prime}, t\right)= & \int_{-\infty}^{\infty} \int_{-\infty}^{\infty} \hat{\omega}^{(m)}\left(\kappa_{1}, \kappa_{2}\right) \\
& \times e^{i \kappa_{1} r_{1}^{\prime}+i \kappa_{2} r_{2}^{\prime}} d \kappa_{1} d \kappa_{2},
\end{aligned}
$$

(36) may be expressed in the form (see Saffman and
Pullin $^{2}$ and Pullin and Saffman ${ }^{7}$ Appendix B, for an analysis of this procedure)

$$
\begin{aligned}
W_{i j}(\boldsymbol{\rho})= & \frac{1}{2 L^{3}} \sum_{m} l_{m} \int_{-\infty}^{\infty} \int_{-\infty}^{\infty} \int_{0}^{\pi} \int_{0}^{2 \pi} \int_{0}^{2 \pi} \\
& \times\left|\hat{\omega}^{(m)}\left(\kappa_{1}, \kappa_{2}, t\right)\right|^{2} e^{-i \kappa_{1} \rho_{1}^{\prime}-i \kappa_{2} \rho_{2}^{\prime} E_{3 i} E_{3 j} P(\alpha, \beta, \gamma)} \\
& \times d \kappa_{1} d \kappa_{2} \sin \alpha d \alpha d \beta d \gamma .
\end{aligned}
$$

Using

$$
\kappa_{1} \rho_{1}^{\prime}+\kappa_{2} \rho_{2}^{\prime}=\left(E_{1 q} \kappa_{1}+E_{2 q} \kappa_{2}\right) \rho_{q},
$$

in (38), and substituting this equation into (29) and the result into (34), gives

$$
\begin{aligned}
& \Theta_{i j}\left(k_{3}\right)=\frac{1}{16 \pi^{3} L^{3}} \sum_{m} l_{m} \int_{-\infty}^{\infty} \int_{-\infty}^{\infty} \int_{-\infty}^{\infty} \int_{-\infty}^{\infty} \int_{-\infty}^{\infty} \int_{-\infty}^{\infty} \int_{-\infty}^{\infty} \int_{0}^{\pi} \int_{0}^{2 \pi} \int_{0}^{2 \pi}\left|\hat{\omega}^{(m)}\left(\kappa_{1}, \kappa_{2}, t\right)\right|^{2}
\end{aligned}
$$

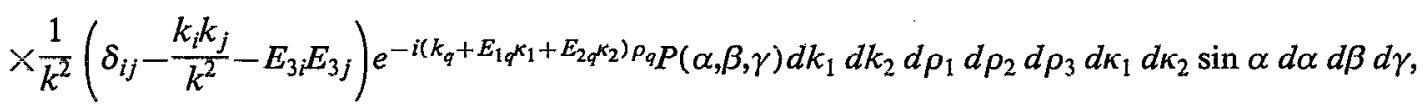

where we have used $E_{3 q} E_{3 q}=1$. The summation over $q$ in the exponential of (40) is noted.

The integrations with respect to $\rho_{1}, \rho_{2}$, and $\rho_{3}$ may be performed by making use of

$$
\frac{1}{2 \pi} \int_{-\infty}^{\infty} e^{-i\left(k_{1}+K\right) \rho_{1}} d \rho_{1}=\delta\left(k_{1}+K\right), \text { etc. }
$$

giving factors of the form $\delta\left(k_{q}+E_{1 q} \kappa_{1}+E_{2 q} \kappa_{2}\right)$ for $q=1,2,3$. This then allows integration with respect to $k_{1}, k_{2}$ to give

$$
\begin{aligned}
\Theta_{i j}\left(k_{3}\right)= & \frac{1}{2 L^{3}} \sum_{m} l_{m} \int_{-\infty}^{\infty} \int_{-\infty}^{\infty} \int_{0}^{\pi} \int_{0}^{2 \pi} \int_{0}^{2 \pi} \\
& \times\left|\hat{\omega}^{(m)}\left(\kappa_{1}, \kappa_{2}, t\right)\right|^{2} \frac{1}{k^{2}}\left(\delta_{i j}-\frac{k_{i} k_{j}}{k^{2}}-E_{3 i} E_{3 j}\right) \\
& \times \delta\left(k_{3}+E_{13} \kappa_{1}+E_{23} \kappa_{2}\right) \\
& \times P(\alpha, \beta, \gamma) d \kappa_{1} d \kappa_{2} \sin \alpha d \alpha d \beta d \gamma
\end{aligned}
$$

where

$$
k_{1}+E_{11} \kappa_{1}+E_{21} \kappa_{2}=0, \quad k_{2}+E_{12} \kappa_{1}+E_{22} \kappa_{2}=0 .
$$

\section{B. Independence of the spin angle $\gamma$}

A significant simplification results when $P$ is independent of the spin angle $\gamma$. In this case the integral with respect to $\gamma$ can be performed by using the relation ${ }^{2}$

$$
\delta[f(x)]=\frac{1}{f^{\prime}\left(x_{0}\right)} \delta\left(x-x_{0}\right)
$$

where $f\left(x_{0}\right)=0$. This gives

$$
\begin{aligned}
\int_{-\infty}^{\infty} & \int_{-\infty}^{\infty} \int_{0}^{2 \pi} \delta\left(k_{3}+E_{13} \kappa_{1}+E_{23} \kappa_{2}\right) \\
\times & F\left(\kappa_{1}, \kappa_{2}, \gamma\right) d \kappa_{1} d \kappa_{2} d \gamma \\
= & \sum_{q=1}^{2} \frac{1}{\sin \alpha} \\
& \times \int_{0}^{2 \pi} \int_{\left|k_{3} / \sin \alpha\right|}^{\infty} \frac{F\left(\kappa \cos \theta_{k}, \kappa \sin \theta_{k}, \gamma_{q}\right) \kappa d \kappa d \theta_{k}}{\left(\kappa^{2}-k_{3}^{2} / \sin ^{2} \alpha\right)^{1 / 2}}
\end{aligned}
$$

where $\gamma_{1}$ and $\gamma_{2}=2 \pi-\left(2 \theta_{k}+\gamma_{1}\right)$ are the two roots of

$$
k_{3} / \sin \alpha=\kappa \cos \left(\gamma_{q}+\theta_{k}\right)
$$

in the range of integration, and where we have put $\kappa_{1}=\kappa \cos \theta_{k}, \kappa_{2}=\kappa \sin \theta_{k}$. We note that from (43), (46) and (2) we may obtain the useful simplifying result $k^{2}=\kappa^{2}$, independent of the Euler angles.

The six independent components of $\Theta_{i j}$ may now be calculated by using (43) and (45)-(46) in (42) and by making use of (2) for the components of $E_{i j}$. We give an intermediate result for only one case, $i=j=3$ for which we obtain, after some lengthy algebra requiring care in evaluating the sum over the roots of (46), the longitudinal onedimensional energy spectrum in the form 
$\Theta_{33}\left(k_{3}\right)=\frac{1}{L^{3}} \sum_{m} l_{m} \int_{0}^{2 \pi} \int_{0}^{\pi} \int_{0}^{2 \pi} \int_{\left|k_{3} / \sin \alpha\right|}^{\infty} \frac{1}{\kappa^{3}}$

$\times\left|\hat{\omega}^{(m)}\left(\kappa \cos \theta_{k}, \kappa \sin \theta_{k}, t\right)\right|^{2}\left(\kappa^{2}-\frac{k_{3}^{2}}{\sin ^{2} \alpha}\right)^{1 / 2}$

$\times \hat{P} \sin ^{2} \alpha d \theta_{k} d \alpha d \beta d \kappa$.

Using the result for the shell-summed energy spectrum, ${ }^{6}$

$E(\kappa)=\frac{2 \pi^{2}}{L^{3}} \sum_{m} l_{m} \int_{0}^{2 \pi} \frac{1}{\kappa}\left|\hat{\omega}^{(m)}\left(\kappa \cos \theta_{k}, \kappa \sin \theta_{k}, t\right)\right|^{2} d \theta_{k}$,

this may be expressed as

$$
\begin{aligned}
\Theta_{33}\left(k_{3}\right)= & \frac{1}{2 \pi^{2}} \int_{0}^{\pi} \int_{0}^{2 \pi} \int_{\left|k_{3} / \sin \alpha\right|}^{\infty} \frac{E(\kappa)}{\kappa^{2}} \\
& \times\left(\kappa^{2}-\frac{k_{3}^{2}}{\sin ^{2} \alpha}\right)^{1 / 2} \hat{P} \sin ^{2} \alpha d \alpha d \beta d \kappa
\end{aligned}
$$

An essential part of the argument is the observation that $E(\kappa)$ given by (48) is independent of $P$ (Refs. 2, 6, and 7).

Results for the other components of the symmetrical tensor $\Theta_{i j}\left(k_{3}\right)$ are

$$
\begin{aligned}
\Theta_{11}\left(k_{3}\right)= & \frac{1}{2 \pi^{2}} \int_{0}^{\pi} \int_{0}^{2 \pi} \int_{\left|k_{3} / \sin \alpha\right|}^{\infty} \frac{E(\kappa)}{\kappa^{2}}\left[\cos ^{2} \alpha\right. \\
& \times \cos ^{2} \beta\left(\kappa^{2}-\frac{k_{3}^{2}}{\sin ^{2} \alpha}\right)^{1 / 2} \\
& \left.+\sin ^{2} \beta \frac{k_{3}^{2}}{\sin ^{2} \alpha}\left(\kappa^{2}-\frac{k_{3}^{2}}{\sin ^{2} \alpha}\right)^{-1 / 2}\right] \hat{P} d \alpha d \beta d \kappa,
\end{aligned}
$$

$$
\begin{aligned}
\Theta_{22}\left(k_{3}\right)= & \frac{1}{2 \pi^{2}} \int_{0}^{\pi} \int_{0}^{2 \pi} \int_{\left|k_{3} / \sin \alpha\right|}^{\infty} \frac{E(\kappa)}{\kappa^{2}}\left[\cos ^{2} \alpha\right. \\
& \times \sin ^{2} \beta\left(\kappa^{2}-\frac{k_{3}^{2}}{\sin ^{2} \alpha}\right)^{1 / 2} \\
& \left.+\cos ^{2} \beta \frac{k_{3}^{2}}{\sin ^{2} \alpha}\left(\kappa^{2}-\frac{k_{3}^{2}}{\sin ^{2} \alpha}\right)^{-1 / 2}\right] \hat{P} d \alpha d \beta d \kappa
\end{aligned}
$$

$$
\begin{aligned}
\Theta_{12}\left(k_{3}\right)= & \frac{1}{4 \pi^{2}} \int_{0}^{\pi} \int_{0}^{2 \pi} \int_{\left|k_{3} / \sin \alpha\right|}^{\infty} \frac{E(\kappa)}{\kappa^{2}} \\
& \times\left[\cos ^{2} \alpha\left(\kappa^{2}-\frac{k_{3}^{2}}{\sin ^{2} \alpha}\right)^{1 / 2}\right. \\
& \left.-\frac{k_{3}^{2}}{\sin ^{2} \alpha}\left(\kappa^{2}-\frac{k_{3}^{2}}{\sin ^{2} \alpha}\right)^{-1 / 2}\right] \hat{P} \sin 2 \beta d \alpha d \beta d \kappa,
\end{aligned}
$$

$\Theta_{13}\left(k_{3}\right)$

$$
\begin{aligned}
= & -\frac{1}{4 \pi^{2}} \int_{0}^{\pi} \int_{0}^{2 \pi} \int_{\left|k_{3} / \sin \alpha\right|}^{\infty} \frac{E(\kappa)}{\kappa^{2}}\left(\kappa^{2}-\frac{k_{3}^{2}}{\sin ^{2} \alpha}\right)^{1 / 2} \\
& \times \hat{P} \sin 2 \alpha \cos \beta d \alpha d \beta d \kappa
\end{aligned}
$$

$\Theta_{23}\left(k_{3}\right)$

$$
\begin{aligned}
= & -\frac{1}{4 \pi^{3}} \int_{0}^{\pi} \int_{0}^{2 \pi} \int_{\left|k_{3} / \sin \alpha\right|}^{\infty} \frac{E(\kappa)}{\kappa^{2}}\left(\kappa^{2}-\frac{k_{3}^{2}}{\sin ^{2} \alpha}\right)^{1 / 2} \\
& \times \hat{P} \sin 2 \alpha \sin \beta d \alpha d \beta d \kappa .
\end{aligned}
$$

The components $\Theta_{11}\left(k_{3}\right), \Theta_{22}\left(k_{3}\right)$ give the lateral onedimensional energy spectra in the 1 and 2 directions, respectively, while the off-diagonal components give the spectra of the cross-components of velocity.

Equations (49)-(54) cannot be further reduced without information on $\hat{P}(\alpha, \beta)$ and $E(\kappa)$. Isotropy corresponds to $P=1$, and it is then clear that $\beta$ integration gives $\Theta_{i j}\left(k_{3}\right)=0, i \neq j$. For this case the $\alpha$ integration in the diagonal components can be performed by first introducing the transformation $u=k_{3} / \sin \alpha$ followed by an exchange of the limits of integration in the $\kappa-u$ plane (see Saffman and Pullin, ${ }^{2}$ Appendix B, for further details). This gives

$$
\begin{aligned}
& \Theta_{11}\left(k_{3}\right)=\Theta_{22}\left(k_{3}\right)=\frac{1}{4} \int_{k_{3}}^{\infty} \frac{E(\kappa)}{\kappa}\left(1+\frac{k_{3}^{2}}{\kappa^{2}}\right) d \kappa, \\
& \Theta_{33}\left(k_{3}\right)=\frac{1}{2} \int_{k_{3}}^{\infty} \frac{E(\kappa)}{\kappa}\left(1-\frac{k_{3}^{2}}{\kappa^{2}}\right) d \kappa,
\end{aligned}
$$

as required (see Batchelor, ${ }^{1}$ Sec. 3.4).

The Reynolds-stress tensor for arbitrary $\hat{P}(\alpha, \beta)$ may be obtained by substitution of (49)-(54) into the first of (32). The $k_{3}$ integration can be performed by exchanging the limits of integration in the $k_{3}-\kappa$ plane, and by making the substitution $k_{3}=\xi \kappa \sin \alpha$ in the $k_{3}$ integral. On performing the $\xi$ integrations, the resulting integrands as functions of $\alpha, \beta$ for $i=1,2,3 ; j=1,2,3$ are found to be proportional to the corresponding components of (7), and so, noting that $\hat{P}$ is independent of $\gamma$, the final expression for the Reynolds stresses may be written in tensor form as

$$
R_{i j}(0)=\overline{\overline{u_{i} u_{j}}}=2 \int_{0}^{\infty} E(\kappa) d \kappa\left\langle E_{p i} Z_{p q} E_{q j}\right\rangle
$$

which agrees with (8). In Appendix A, an alternative derivation of (57) is given. 


\section{Some example calculations}

If $E(\kappa)$ exhibits power-law behavior in some range of wave numbers, (49)-(54) can be expressed in more compact form. We assume that there exists an inertial range in which $E$ takes the standard form, from (13),

$$
E(\kappa)=\mathscr{K}_{0} \epsilon^{2 / 3} \kappa^{-5 / 3}, \frac{1}{L}<\kappa<\frac{1}{\eta},
$$

where $L$ is the length scale of the large eddies. When (58) is used in (49)-(54), the $\kappa$ integrations can be reduced to scaling numbers by the transformation $\kappa=\eta k_{3} / \sin \alpha$, and it is found that

$$
\Theta_{i j}\left(k_{3}\right)=\mathscr{K}_{0} \epsilon^{2 / 3} k_{3}^{-5 / 3}\left(\frac{\Gamma\left[\frac{5}{6}\right]}{2 \pi^{1 / 2} \Gamma\left[\frac{7}{3}\right]} A_{i j}\right),
$$

where the angle bracket in (59) refers to (4). We remark that the $k_{3}^{-5 / 3}$ form of $(59)$ depends on the pure $\kappa^{-5 / 3}$ power law in (58). When $\hat{P}=1$ the bracket in (59) may be calculated, and is found to be diagonal with elements $\left(\frac{12}{55}\right.$, $\left.\frac{12}{55,55}\right)$, as required for the one-dimensional longitudinal and lateral spectra of isotropic turbulence. This may be verified by using $(58)$ in $(55)-(56)$.

While we emphasize that in the large-eddy simulation models discussed in Sec. IV, $\hat{P}(\alpha, \beta)$ is determined by the action of large scales, we nevertheless show, for the purposes of illustration, a calculation for a special choice of $\hat{P}$. We take, as an example, a distribution of Euler angles uniform on the half-cone $\alpha=\alpha_{0}=\pi / 4,3 \pi / 2<\beta<2 \pi, 0<\beta$ $<\pi / 2$. This corresponds to a $\hat{P}$ given by

$$
\hat{P}(\alpha, \beta)=\left\{\begin{array}{l}
\frac{4 \delta\left(\alpha-\alpha_{0}\right)}{\sin \alpha}, \frac{3 \pi}{2} \leqslant \beta<2 \pi, 0<\beta<\frac{\pi}{2} \\
0, \quad \frac{\pi}{2} \leqslant \beta<\frac{3 \pi}{2} .
\end{array}\right.
$$

This choice is perhaps typical of the legs of lambda vortices in a turbulent boundary-layer flow for which the mean flow is in the $r_{3}$ direction with $r_{1}$ normal to the wall (which lies in the $r_{2}-r_{3}$ plane) and $r_{2}$ in the spanwise direction. The angle bracket in (59) can then easily be calculated to be

$$
\begin{aligned}
& \left\langle\frac{\Gamma\left(\frac{5}{6}\right)}{2 \pi^{1 / 2} \Gamma\left(\frac{7}{3}\right)} A_{i j}\right\rangle \\
& \quad=\left(\begin{array}{ccc}
\frac{13}{6} 2^{-4 / 3} & 0 & -(1 / \pi) 2^{-1 / 3} \\
0 & \frac{13}{6} 2^{-4 / 3} & 0 \\
-(1 / \pi) 2^{-1 / 3} & 0 & 2^{-4 / 3}
\end{array}\right),
\end{aligned}
$$

while, from (4) and (7) and (8), the Reynolds-stress tensor is

$$
R_{i j}(0)=\overline{\overline{u_{m} u_{m}}}\left(\begin{array}{ccc}
\frac{3}{8} & 0 & -1 / 2 \pi \\
0 & \frac{3}{8} & 0 \\
-1 / 2 \pi & 0 & \frac{1}{4}
\end{array}\right) .
$$

From (63), the ratio $\overline{\overline{u_{1} u_{3}}} \overline{\overline{u_{m} u_{m}}}=-1 / 2 \pi \approx-0.159$. It is well known that this ratio is approximately constant over much of a zero-pressure gradient boundary layer with a value (Hinze, ${ }^{13}$ Figs. 7-22) of near -0.16 . If in place of (61) we assume that all vortex structures are aligned with $\alpha=\pi / 4, \beta=0$, then this ratio is presently calculated as -0.25 .

\section{CONCLUDING REMARKS}

We have considered a vortex model of homogeneous turbulence that allows for statistically anisotropic properties of the flow. The model is based on the assumption of vortex structures, with general properties like those of the Lundgren-Townsend models, the anisotropy being incorporated by the probability density functions of the orientations of the structures relative to axes fixed in space. A simple result is obtained when the structures have circularly symmetric statistics, that is, the probability distribution of the Euler angles is independent of the spin angle $\gamma$, which relates the Reynolds stresses (i.e., the covariances of perpendicular velocity components) to the turbulent kinetic energy and averages of the vortex orientation. This allows identifying the contributions to the Reynolds stress from different scales and suggests a subgrid Reynoldsstress model for the evolution of large scales. An illustrative example that may be appropriate for boundary-layer turbulence strained by a simple shear predicts values of the ratio of Reynolds stress to mean turbulent kinetic energy, in reasonable accord with experimental data.

It is also shown that the formulation leads to integral expressions for the one-dimensional velocity covariance spectra in terms of the probability density functions and the shell-summed energy spectrum. Comparison with ex- 
perimental data, when it becomes available, would allow inferences to be made about the anisotropic nature of the vortex structures.

\section{ACKNOWLEDGMENTS}

DIP was partially supported by National Science Foundation Grant No. CTS-9311811 and PGS was par- tially supported by the Department of Energy under Grant No. DE-FG03-89ER25073. The authors have benefited from discussions with Dr. A. Leonard.

\section{APPENDIX A: DIRECT CALCULATION OF $R_{i j}(0)$}

Substituting (40) into the first of (32) gives

$$
\begin{aligned}
& R_{i j}(0)=\frac{1}{16 \pi^{3} L^{3}} \sum_{m} l_{m} \int_{-\infty}^{\infty} \int_{-\infty}^{\infty} \int_{-\infty}^{\infty} \int_{-\infty}^{\infty} \int_{-\infty}^{\infty} \int_{-\infty}^{\infty} \int_{-\infty}^{\infty} \int_{-\infty}^{\infty} \int_{0}^{\pi} \int_{0}^{2 \pi} \int_{0}^{2 \pi}\left|\hat{\omega}^{(m)}\left(\kappa_{1}, \kappa_{2}, t\right)\right|^{2}
\end{aligned}
$$

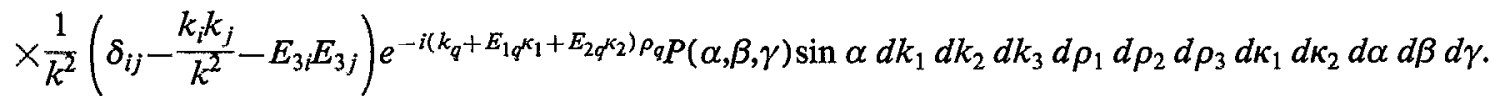

The integrations with respect to $\rho_{1}, \rho_{2}$, and $\rho_{3}$ may be performed by making use of (41), giving factors $\delta\left(k_{q}+E_{1 q} \kappa_{1}+E_{2 q} \kappa_{2}\right), q=1,2,3$. After integration with respect to $k_{1}, k_{2}$, and $k_{3}$, and following some algebra, we obtain

$$
\begin{aligned}
R_{i j}(0)= & Q_{0} \delta_{i j}-Q_{1}\left\langle\left\langle E_{3 i} E_{3 j}\right\rangle\right\rangle-Q_{2}\left\langle\left\langle E_{1 i} E_{1 j}\right\rangle\right\rangle \\
& -Q_{3}\left\langle\left\langle E_{1 i} E_{2 j}+E_{2 i} E_{1 j}\right\rangle\right\rangle-Q_{4}\left\langle\left\langle E_{2 i} E_{2 j}\right\rangle\right\rangle, \\
Q_{0}= & Q_{1}=\frac{4 \pi^{2}}{L^{3}} \sum_{m} l_{m} \int_{0}^{\infty} \frac{1}{\kappa} \int_{0}^{2 \pi} \\
& \times\left|\hat{\omega}^{(m)}\left(\kappa \cos \theta_{k}, \kappa \sin \theta_{k}, l\right)\right|^{2} d \kappa d \theta_{k}, \\
Q_{2}= & \frac{4 \pi^{2}}{L^{3}} \sum_{m} l_{m} \int_{0}^{\infty} \frac{1}{\kappa} \int_{0}^{2 \pi}\left|\hat{\omega}^{(m)}\left(\kappa \cos \theta_{k}, \kappa \sin \theta_{k}, t\right)\right|^{2} \\
& \times \cos ^{2} \theta_{k} d \theta_{k} d \kappa, \\
Q_{3}= & \frac{4 \pi^{2}}{L^{3}} \sum_{m} l_{m} \int_{0}^{\infty} \frac{1}{\kappa} \int_{0}^{2 \pi}\left|\hat{\omega}^{(m)}\left(\kappa \cos \theta_{k}, \kappa \sin \theta_{k}, t\right)\right|^{2} \\
& \times \sin \theta_{k} \cos \theta_{k} d \theta_{k} d \kappa, \\
Q_{4}= & \frac{4 \pi^{2}}{L^{3}} \sum_{m} l_{m} \int_{0}^{\infty} \frac{1}{\kappa} \int_{0}^{2 \pi}\left|\hat{\omega}^{(m)}\left(\kappa \cos \theta_{k}, \kappa \sin \theta_{k}, t\right)\right|^{2} \\
& \times \sin ^{2} \theta_{k} d \kappa d \theta_{k},
\end{aligned}
$$

where $\kappa_{1}=\kappa \cos \theta_{k}$ and $\kappa_{1}=\kappa \sin \theta_{k}$. The integrals may be treated using a method developed by Lundgren ${ }^{6}$ (Sec. III B) for calculation of the energy spectrum. Briefly, a transformation to polar coordinates in the $\left(r_{1}^{\prime}-r_{2}^{\prime}\right)$ plane is made with $r_{1}^{\prime}=r^{\prime} \cos \theta, r_{2}^{\prime}=r^{\prime} \sin \theta$, and the first of (37) is written as

$$
\begin{aligned}
\hat{\omega}^{(m)}\left(\kappa_{1}, \kappa_{2}\right)= & \frac{1}{4 \pi^{2}} \int_{0}^{\infty} \int_{0}^{2 \pi} \\
& \times \omega^{(m)}\left(r^{\prime}, \theta, t\right) e^{-i \kappa r^{\prime} \cos \left(\theta-\theta_{k}\right)} r^{\prime} d r^{\prime} d \theta .
\end{aligned}
$$

Next, $\omega^{(m)}\left(r^{\prime}, \theta, t\right)$ is expanded as a Fourier series,

$$
\omega^{(m)}\left(r^{\prime}, \theta, t\right)=\sum_{-\infty}^{\infty} \omega_{n}^{(m)}\left(r^{\prime}, t\right) e^{i n \theta} .
$$

When (A5) is substituted into (A4) and use is made of the result

$$
\int_{0}^{2 \pi} e^{i n \theta-i \kappa r^{\prime} \cos \left(\theta-\theta_{k}\right)} d \theta=(-i)^{n} 2 \pi J_{n}\left(\kappa r^{\prime}\right) e^{i n \theta_{k}}
$$

where $J_{n}$ is the Bessel function, the integrations with respect to both $\theta$ and $\theta_{k}$ in (A18) can be performed, and it is found that

$$
\begin{aligned}
& Q_{0}=Q_{1}=2 \int_{0}^{\infty} E(\kappa) d \kappa, \\
& Q_{2}=\int_{0}^{\infty} E(\kappa) d \kappa-\int_{0}^{\infty} F(\kappa) d \kappa, \\
& Q_{3}=\int_{0}^{\infty} G(\kappa) d \kappa, \quad Q_{4}=\int_{0}^{\infty} E(\kappa) d \kappa+\int_{0}^{\infty} F(\kappa) d \kappa,
\end{aligned}
$$

where

$$
\begin{aligned}
& E(\kappa)=\frac{\pi}{\kappa L^{3}} \sum_{m} l_{m}\left(\left|I_{0}^{(m)}(\kappa)\right|^{2}+\sum_{n=1}^{\infty}\left|I_{n}^{(m)}(\kappa)\right|^{2}\right), \\
& F(\kappa)=\frac{\pi}{\kappa L^{3}} \sum_{m} l_{m} \sum_{n=-\infty}^{\infty} \Re\left[I_{n}^{(m)}(\kappa) I_{n-2}^{(m)^{*}}(\kappa)\right], \\
& G(\kappa)=\frac{\pi}{\kappa L^{3}} \sum_{m} l_{m} \sum_{n=-\infty}^{\infty} \Re\left[I_{n}^{(m)}(\kappa) I_{n-2}^{(m)^{*}}(\kappa)\right],
\end{aligned}
$$

and

$$
I_{n}^{(m)}(\kappa)=\int_{0}^{\infty} J_{n}\left(\kappa r^{\prime}\right) \omega_{n}^{(m)}\left(r^{\prime}, t\right) r^{\prime} d r^{\prime},
$$

where $E(\kappa)$ is the shell-summed energy spectrum and * denotes the complex conjugate. An expression for $R_{i j}(0)$ is obtained when (A7) and (A8) is substituted into (A2).

When $P$ is independent of the spin angle $\gamma$, we can replace the double angle brackets in (A2) by single angle 
brackets in the sense of (3)-(4). The integrals $\left\langle E_{1 i} E_{1 j}-E_{2 i} E_{2 j}\right\rangle$ and $\left\langle E_{1 i} E_{2 j}+E_{2 i} E_{1 j}\right\rangle$, which arise when (A7) are used in (A2) are then zero, and we obtain

$$
\begin{aligned}
R_{i j}(0)= & 2 \int_{0}^{\infty} E(\kappa) d \kappa\left\langle\delta_{i j}-\frac{1}{2}\left(E_{1 i} E_{1 j}+E_{2 i} E_{2 j}\right.\right. \\
& \left.\left.+2 E_{3 i} E_{3 j}\right)\right\rangle .
\end{aligned}
$$

Note that there is now no dependency on $F(\kappa)$ and $G(\kappa)$. The expression inside the angle brackets in (A10) may be shown to be equal to $E_{p i} Z_{p q} E_{q j}$, and we finally have

$$
R_{i j}(0)=\overline{\overline{u_{i} u_{j}}}=2 \int_{0}^{\infty} E(\kappa) d \kappa\left\langle E_{p i} Z_{p q} E_{q j}\right\rangle,
$$

which agrees with (8) and with (57).

\section{APPENDIX B: ANALYSIS OF THE SUBGRID STRESSES}

Here we investigate the behavior of the model given by (15), (17), and (22)-(23) in the limits where $\eta$ is both small and large compared with $\Delta x$. For simplicity, we consider the case when only $\lambda_{3}$ is positive. Using (23) (with $\lambda_{2}$ replaced by zero) in (17) gives

$$
\begin{aligned}
\epsilon= & 2 v\left(\lambda_{1}^{2}+\lambda_{2}^{2}+\lambda_{3}^{2}\right)+C^{2 / 3} \mathscr{K}_{0} \epsilon^{1 / 2} v^{1 / 2} \lambda_{3} \\
& \times \Gamma\left(-\frac{2}{3}, C k_{c}\left(v^{3} / \epsilon\right)^{1 / 4}\right),
\end{aligned}
$$

where we have replaced $\eta$ by $\left(v^{3} / \epsilon\right)^{1 / 4}$. With $C, \mathscr{K}_{0}$, and $v$ given, $k_{c}$ fixed by the cell size $\Delta x$, and $\left(\lambda_{1}, \lambda_{2}, \lambda_{3}\right)$ given by the instantaneous supergrid flow, (B1) can be solved for $\epsilon$ in each cell, which then defines the local Kolmogorov length $\eta=\left(v^{3} / \epsilon\right)^{1 / 4}$. Our interest is the behavior of the model when $k_{c} \eta<1$, and $k_{c} \eta>1$, respectively. We first make the transformations to dimensionless variables,

$$
X=\frac{k_{c}^{2} \epsilon}{\lambda_{3}^{3}}, \quad Y=k_{c} \eta,
$$

under which (B1) becomes

$X^{2 / 3}-2 B Y^{4 / 3}-C^{2 / 3} \mathscr{K}_{0} X^{1 / 3} Y^{2 / 3} \Gamma\left(-\frac{2}{3}, C Y\right)=0$,

where

$$
B=\left(\frac{\lambda_{1}}{\lambda_{3}}\right)^{2}+\left(\frac{\lambda_{2}}{\lambda_{3}}\right)^{2}+1
$$

In what follows it is assumed that $B$ is of order unity.

We first consider $Y \rightarrow 0$, i.e., $\eta<\Delta x$. Since for $C Y<1$ we have

$$
\Gamma\left(\frac{-2}{3}, C Y\right)=\frac{3}{2 C^{2 / 3}} Y^{-2 / 3}+O(1),
$$

then it is clear that the solution to (B3) is given to leading order by a balance between the first and third terms, giving

$$
X=\frac{27 \mathscr{K}_{0}^{3}}{8} \text { or } \epsilon=\frac{27 \mathscr{K}_{0}^{3} \lambda_{3}^{3}}{8 k_{c}^{2}},
$$

which is equivalent to (19). From (15), it follows that the ratio

$$
\frac{\left\|T_{i j}\right\|}{v\left\|\widetilde{S}_{i j}\right\|}=O\left(\frac{\lambda_{3}}{k_{c}^{2} v}\right)
$$

where $\|\cdots\|$ refers to the component of maximum magnitude and $\lambda_{3} / k_{c}^{2} v$ is the effective supergrid cell Reynolds number. When this quantity is large (B7) shows that the subgrid stresses are dominant.

Next, consider $Y$ large, i.e., $\eta>\Delta x$. The incomplete gamma function is then

$$
\Gamma\left(-\frac{2}{3}, C Y\right) \sim(C Y)^{-5 / 3} e^{-C Y},
$$

and the leading-order solution to (B3) is determined by a balance between the first and second terms. Thus

$$
X^{2 / 3}=2 B Y^{4 / 3} \text { or } \epsilon=2 \nu\left(\lambda_{1}^{2}+\lambda_{2}^{2}+\lambda_{3}^{2}\right),
$$

so that the dissipation is dominated by the supergrid stresses. A short calculation then shows that

$$
\frac{\left\|T_{i j}\right\|}{v\left\|\widetilde{S}_{i j}\right\|}=O\left\{\left(\frac{\lambda_{3}}{k_{c}^{2} v}\right)^{5 / 6} \exp \left[-\frac{C}{(2 B)^{1 / 4}}\left(\frac{\lambda_{3}}{k_{c}^{2} v}\right)^{-1 / 2}\right]\right\} \text {, }
$$

which is small when $\lambda_{3} / k_{c}^{2} v$ is small. Therefore the model reverts to the fully resolved Navier-Stokes equations in this limit.

${ }^{1} \mathrm{G}$. K. Batchelor, The Theory of Homogeneous Turbulence (Cambridge University Press, Cambridge, 1953).

${ }^{2}$ P. G. Saffman and D. I. Pullin, "Anisotropy of the LundgrenTownsend model of fine-scale turbulence," Phys. Fluids A 6, 802 (1994).

${ }^{3}$ R. M. Kerr, "Higher-order derivative correlations and the alignment of small-scale structures in isotropic numerical turbulence," J. Fluid Mech. 153, 31 (1985). See also NASA Tech. Memo. TM 84407 (1983).

${ }^{4}$ W. T. Ashurst, A. R. Kerstein, R. M. Kerr, and C. H. Gibson, "Alignment of vorticity and scalar-gradient in simulated Navier-Stokes turbulence," Phys. Fluids 30, 2343 (1987).

${ }^{5} \mathrm{~A}$. Vincent and M. Meneguzzi, "The spatial structure and statistical properties of homogeneous turbulence," J. Fluid Mech. 225, 1 (1991).

${ }^{6} \mathrm{~T}$. S. Lundgren, "Strained spiral vortex model for turbulent fine structure," Phys. Fluids 25, 2193 (1982).

${ }^{7}$ D. I. Pullin and P. G. Saffman, "On the Lundgren-Townsend model of turbulent fine scales," Phys. Fluids A 5, 126 (1993)

${ }^{8} \mathrm{~J}$. M. Burgers, "A mathematical model illustrating the theory of turbulence," Adv. Appl. Mech. 1, 171 (1948).

${ }^{9}$ H. Jeffreys and B. Jeffreys, Methods of Mathematical Physics (Cambridge University Press, Cambridge, 1950).

${ }^{10} \mathrm{~A}$. Leonard, "Energy cascade in large-eddy simulations of turbulent fluid flows," Advances in Geophysics, edited by F. N. Frankiel and R. E. Munn (Academic, New York, 1974), Vol. 18A, pp. 237-248.

${ }^{11}$ M. Germano, U. Piomelli, P. Moin, and W. H. Cabot, "A dynamic subgrid-scale eddy viscosity model," Phys. Fluids A 3, 1760 (1991).

${ }^{12}$ M. S. Chong, A. E. Perry, and B. J. Cantwell, "A general classification of three-dimensional flow fields," Phys. Fluids A 2, 765 (1990).

${ }^{13}$ J. O. Hinze, Turbulence, 2nd ed. (McGraw-Hill, New York, 1975). 